\title{
Quantized Radiation States from the Infinite Dicke Model
}

\author{
By \\ Reinhard HoNEgGER* and Alfred RIECKERs**
}

\begin{abstract}
By interpreting infinitely many two-level atoms as a mean field quantum lattice system in a recent paper the time evolution of the Dicke Maser model has been elaborated in terms of operator algebraic methods. Using these results, here the emitted radiation of the infinite Dicke model is investigated. It is shown how the collective behaviour of the atoms influences the quantized radiation, which for large times becomes classically coherent (in the sense of Glauber). The field modes which are (approximately) resonant with the level-splitting energy of the atoms are found to be the essential part of the generated coherent light, and thereby determine its macroscopic nature. Furthermore, the destruction and revival of coherence, the mean number of the emitted photons during the time evolution, as well as their spatial distribution are discussed.
\end{abstract}

\section{$\S 1$. Introduction}

In terms of operator algebraic quantum statistical mechanics the present work gives a detailed microscopic description how the coherent light is dynamically generated from the macroscopic preparation of the radiating atoms or molecules. For this we use the extended Dicke model of an earlier paper [1] in which we studied the limiting dynamics with the number of the radiating two-level atoms going to infinity. Thereby the interaction strength remains on the level of finitely many atoms, which one may interpret as a weak coupling of the radiation field to a macroscopic atomic reservoir.

In terms of a perturbational treatment one first selects adequate representations of the uncoupled systems. The representation of the atomic $C^{*}$-algebra is chosen according to the sharp values of the cooperation and excitation numbers, $\eta$ resp. $\gamma$, which (up to a phase) completely determine and characterize the classical part, that is the collective features of the

Communicated by H. Araki, June 10, 1992.

1991 Mathematics Subject Classification: 46L60, 81V80.

* Institut für Theoretische Physik, Universität Tübingen, 72076 Tübingen, Germany.

** Institut für Theoretische Physik, Universität Tübingen, 72076 Tübingen, Germany. 
infinite atomic system due to its macroscopic preparation.

In [1] the photons had testfunctions of unspecified nature. In the present investigation the physically real case is considered. The electromagnetic field is assumed to be quantized in the full euclidean space $\mathbb{R}^{3}$ and all (momentum-) modes $k \in \mathbb{R}^{3}$ are included into the model discussion. However, for notational simplicity in Sections 2 to 4 only one direction of polarization is considered, whereas in Section 5 we briefly outline the case of arbitrary polarization. For the relevant representation for the photonic $C^{*}$-Weyl algebra the Fock representation is chosen, since at time zero only a few photons are assumed to be present. These assumptions are essential for the emitted radiation to become coherent during the time evolution.

The interaction of the atoms and the radiation is given in terms of the so-called coupling function $\phi$. Their Fourier transform $\hat{\phi}$ evaluated at $k \in \mathbb{R}^{3}$, $\hat{\phi}(k)$, gives the coupling constant between each two-level atom (with level-splitting $\varepsilon>0$ ) and the field mode $k$. In physical applications $\phi$ is uniquely calculated by use of the wave functions of the two energy levels of the type of atoms or molecules under consideration. For hydrogen-like systems we give the explicit formula in Section 5. It is based on the rotating-wave-approximation for the interaction (for more details see [2, Section 3.2], [3]).

Our investigations concerning the Dicke model ([4], [1] and the present paper) are inspired by the work of Davies [5], [6], where related problems are discussed, however, by considering for the dynamics only the collective behaviour of the atoms, which there is set up in an ad hoc manner. In [1] the infinite atomic system is regarded as a mean field quantum lattice system, so that in the total limiting dynamics both aspects of the atoms, the quantum as well as the classical part, occure.

The present paper is devoted to the emitted radiation of the infinite Dicke model. If $v_{t}, t \in \mathbb{R}$, is the (limiting) Schrödinger dynamics of the total system, we restrict the time evolved states $v_{t}(\omega)$ for arbitrary (normal in the uncoupled representation) initial states $\omega$ to the photon field, the restriction of which we denote by $\left.v_{t}(\omega)\right|_{b}$.

We perform the infinite time limit $t \rightarrow \infty$ and prove the existence and the form of the time asymptotic photon states $R_{t}(\omega)$ in the sense of

$$
\underset{t \rightarrow \infty}{\text { weak }^{*}-\lim }\left(\left.v_{t}(\omega)\right|_{b}-R_{t}(\omega)\right)=0
$$


The time dependence in $R_{t}(\omega)$ is periodic with period $\frac{2 \pi}{\varepsilon}$ and arises from the classical flow on the classical phase space of the atoms, which expresses their collective dynamical behaviour. From the shape of the states $R_{t}(\omega)$ one recognizes how the classical, macroscopic ordering of the atomic system influences the photon field. The time asymptotic states turn out to be (fully) coherent, classical states on the photonic $C^{*}$-Weyl algebra.

The coherence properties are discussed in their operator algebraic version ([7], [8], [9], [10], [11]), which is an extension and refinement of Glauber's original definition [12] and obtained by a smearing procedure with one-photon testfunctions. The algebraic formulation of a photon state to be coherent is characterized by the factorization of the normally ordered expectation values of the creation and annihilation operators with respect to a linear form on the one-photon testfunction space. This extended coherence condition naturally allows to consider more general cases than coherent states, which are normal to the Fock representation.

Indeed, here in the Dicke model it depends on the coupling constants $\hat{\phi}(k)$ for the resonant modes $k$ (which fulfill $|k|=\varepsilon$ ), whether the generated coherent light is of microscopic or macroscopic nature, that is, whether the appearing coherent states $R_{t}(\omega)$ are normal resp. disjoint to the Fock representation.

As we will see, the coherence properties occurring for large times are determined by a linear form $L$ on the one-photon testfunctions, which depends uniquely on the coupling function $\phi$, and which is additively decomposed into two very different terms. The first one represents the exact resonance between the radiation field and the two levels of each atom, whereas the other term concerns the remaining modes.

Because, as mentioned above, the coupling function $\phi$ is uniquely determined by the wave functions of the considered two energy levels, we have in general $\hat{\phi}(k) \neq 0$ for some resonant modes $k$, which is equivalent for the linear form $L$ to be unbounded with respect to the norm topology on the one-photon testfunction space. As a consequence our coherent time asymptotic states $R_{t}(\omega)$ are not realizable by density operators on Fock space. In fact, for unbounded $L$ during the time evolution there are so many emitted photons, that $\left.v_{t}(\omega)\right|_{b}$ leaves Fock space, when time goes to infinity. The statistical correlations inherent in the factorization property of $R_{t}(\omega)$ then extend over so many photons (the photon mean number is 
strictly infinite) that macroscopic features of the electromagnetic field are generated. Especially a macroscopic phase observable is displayed, which arises from the classical atomic ordering, which is expressed in terms of the fixed cooperation and excitation numbers, $\eta$ resp. $\gamma$, and the collective phase observable. This demonstrates that for unbounded $L$ in the asymptotic states one recovers the classical atomic aspects.

In detail we proceed as follows. In Section 2 we recapitulate the notions and results of [1], as well as some preliminary facts concerning states on the Weyl algebra. The linear form $L$, the time asymptotic states $R_{t}(\omega)$ and their averages $\bar{R}(\omega)$ over the time period are deduced in Section 3. Section 4 is devoted to a discussion of the properties of the emitted radiation: microscopic and macroscopic quantum optical coherence, destruction and revival of coherence, the mean number of emitted photons during the time evolution, and finally the spatial distribution of the photons in the time asymptotic states. As mentioned above, in Section 5 we briefly outline the case of all directions of polarization.

For the comparison of the present model with the more common versions with finitely many atoms we refer to the review paper [13].

\section{$\S 2$. Description of the Model}

The following formulation of the Dicke model is based on the previous paper [1]. For the sake of a self-contained presentation we recapitulate some fundamental notions and results, supplementing some generalities of Boson states.

The atomic system is regarded as an infinite mean field quantum lattice system with the inductive limit $C^{*}$-algebra

$$
\mathscr{A}=\bigotimes_{n \in \mathbb{N}} M_{2}
$$

where the complex $2 \times 2$-matrices $M_{2}$ are the algebra of observables for each two-level atom. Each atom is assumed to have the same Hamiltonian with a strictly positive level-splitting $\varepsilon>0$.

The electromagnetic field is taken to be quantized in the whole euclidean space $\mathbb{R}^{3}$, but for notational simplicity only one direction of polarization is considered. In Section 5, however, we briefly outline the considerations including all directions of polarization. The single photon Hamiltonian 
$\sqrt{-\Delta}$ acts on $\mathrm{L}^{2}\left(\boldsymbol{R}^{3}\right)$ ( $\Delta$ denotes the usual Laplacian on $\boldsymbol{R}^{3}$ ) such that the associated unitary group leaves the one-photon testfunction space $E$ invariant,

$$
\exp \{i t \sqrt{-\Delta}\}(E)=E \quad \forall t \in \boldsymbol{R}
$$

$E$ should be a dense subspace of $\mathrm{L}^{2}\left(\boldsymbol{R}^{3}\right)$ and will be further specified in Section 3. The $C^{*}$-algebra for the photon field is the Weyl algebra $\mathscr{W}(E)$ over $E$ [14].

The representation $\left(\Pi_{a}, \mathscr{H}_{a}\right)$ of $\mathscr{A}$ is chosen according to sharp values of the cooperation and excitation numbers, $\eta$ resp. $\gamma$, which satisfy

$$
\left|\gamma-\frac{1}{2}\right| \leq \frac{\eta}{2}, \quad \eta, \gamma \in[0,1] .
$$

The representation Hilbert space $\mathscr{H}_{a}$ is then a tensor product

$$
\mathscr{H}_{a}=\mathscr{H}_{0} \otimes \mathrm{L}^{2}\left(T_{\eta, \gamma}, \lambda^{\eta, \gamma}\right)
$$

with a separable Hilbert space $\mathscr{H}_{0}$ and the normalized Lebesgue measure $\lambda^{\eta, \gamma}$ on the circle line

$$
T_{\eta, \gamma}=\left\{x \in \boldsymbol{R}^{3} \mid\|x\|=\eta / 2, x_{3}=\gamma-1 / 2\right\}
$$

As in [1] we use the parametrization of $T_{\eta, \gamma}$ with the phase angle $\vartheta \in[0,2 \pi[$, in which $T_{\eta, \gamma}=\left[0,2 \pi\left[\right.\right.$ and $d \lambda^{\eta, \gamma}(\vartheta)=\frac{d \vartheta}{2 \pi}$.

By $\mathscr{F}_{a}$ we denote the folium of the $\Pi_{a}$-normal states on $\mathscr{A}$, which are just the normal states on the von Neumann algebra

$$
\mathscr{M}_{a}=\Pi_{a}(\mathscr{A})^{\prime \prime}=\mathscr{M}_{0} \bar{\otimes} \mathrm{L}^{\infty}\left(T_{\eta, \gamma}, \lambda^{\eta, \gamma}\right),
$$

where $\mathscr{M}_{0}$ is a factor acting on $\mathscr{H}_{0}$.

Assuming to exist at time $t=0$ only a few photons, for the quantized radiation system the Fock representation $\Pi_{F}$ of $\mathscr{W}(E)$ on the Bose-Fock space $F_{+} \equiv F_{+}\left(\mathrm{L}^{2}\left(R^{3}\right)\right)$ is selected.

In the representation $\Pi_{a} \otimes \Pi_{F}$ of the composite system algebra $\mathscr{A} \otimes \mathscr{W}(E)$ the limiting dynamics is unitarily implementable and given in the representation Hilbert space 


$$
\mathscr{H}_{a, F}:=\mathscr{H}_{a} \otimes F_{+}
$$

by the essentially selfadjoint Hamiltonian $(\lambda>0$ is a constant)

$$
H=\underbrace{A \otimes \mathbb{1}_{F}}_{\text {free atoms }}+\underbrace{\mathbb{1}_{a} \otimes H_{b}}_{\text {free photons }}+\underbrace{\lambda\left(B_{-} \otimes a_{F}^{*}(\phi)+B_{+} \otimes a_{F}(\phi)\right)}_{\text {interaction }} .
$$

Here $A$ generates the free atomic (limiting) dynamics, which acts on the center $\mathscr{Z}_{a}=\mathbb{1}_{0} \otimes L^{\infty}\left(T_{\eta, \gamma}, \lambda^{\eta, \gamma}\right)$ of $\mathscr{M}_{a}$ in terms of the flow $\varphi_{t}^{\eta, \gamma}$ on $T_{\eta, \gamma}$,

$$
\varphi_{t}^{\eta, \gamma}(\vartheta)=\vartheta+\varepsilon t \quad \forall t \in \mathbb{R} \quad \forall \vartheta \in\left[0,2 \pi\left[=T_{\eta, \gamma}\right.\right.
$$

by the equation

$$
\exp \{i t A\}\left(\mathbb{1}_{0} \otimes G\right) \exp \{-i t A\}=\mathbb{1}_{0} \otimes G \circ \varphi_{t}^{\eta, \gamma} \quad \forall G \in \mathbb{L}^{\infty}\left(T_{\eta, \gamma}, \lambda^{\eta, \gamma}\right)
$$

In the coupling term $B_{ \pm}=\mathbb{1}_{0} \otimes \beta e^{ \pm i \vartheta} \in \mathscr{Z}_{a}$ are the collective raising and lowering operators of the atoms, where

$$
\beta=\sqrt{(\eta / 2)^{2}-(\gamma-1 / 2)^{2}}
$$

is the radius of the circle line $T_{\eta, \gamma} . \quad H_{b}=\mathrm{d} \Gamma(\sqrt{-\Delta})$ denotes the second quantization of one-photon Hamiltonian $\sqrt{-\Delta} . \quad a_{F}(f), a_{F}^{*}(f), f \in \mathbb{L}^{2}\left(\mathbb{R}^{3}\right)$, are the smeared Fock annihilation and creation operators. The Fourier transform $\hat{\phi}(k)$ of the coupling function $\phi \in \mathbb{L}^{2}\left(\boldsymbol{R}^{3}\right)$ represents the coupling constants between each atom and the field mode $k \in \mathbb{R}^{3}$. In momentum space the operators $H_{b}$ and $a_{F}^{*}(\phi)$ formally are written as $\int|k| a_{k}^{*} a_{k} \mathrm{~d}^{3} k$, resp. $\int \hat{\phi}(k) a_{k}^{*} \mathrm{~d}^{3} k$.

For later reference let us give here the explicit expressions of the unitaries $\mathrm{e}^{i t H}, t \in \mathbb{R}$, which are derived in [1, Theorem 4.3]:

$$
\mathrm{e}^{i t H}=\left[\mathbb{1}_{\mathscr{H}_{0}} \otimes \int_{[0,2 \pi[}^{\oplus} \mathrm{e}^{i \kappa(t)} W_{F}(f(t, \vartheta)) \frac{\mathrm{d} \vartheta}{2 \pi}\right]\left[\mathrm{e}^{i t A} \otimes \mathrm{e}^{i t H_{b}}\right] .
$$

where

$$
\begin{aligned}
\kappa(t) & =-\beta^{2} \lambda^{2} \int_{u=0}^{t} \mathrm{~d} u \int_{v=0}^{u} \mathrm{~d} v \operatorname{Im}\left\langle\mathrm{e}^{i(\sqrt{-\Delta}-\varepsilon) v} \phi \mid \mathrm{e}^{i(\sqrt{-\Delta}-\varepsilon) u} \phi\right\rangle \in \mathbb{R}, \\
f(t, \vartheta) & =\sqrt{2} \beta \lambda \mathrm{e}^{-i v} \int_{u=0}^{t} \mathrm{~d} u \mathrm{e}^{i(\sqrt{-\Delta}-\varepsilon) u} \phi \in \mathrm{L}^{2}\left(R^{3}\right) .
\end{aligned}
$$


Let be $\mathscr{F}_{a, F}$ the folium of $\Pi_{a} \otimes \Pi_{F}$-normal states on $\mathscr{A} \otimes \mathscr{W}(E)$. In our notation we do not distinguish between states in $\mathscr{F}_{a . F}$ and their unique normal extensions to the associated von Neumann algebra

$$
\mathscr{M}_{a, F}=\left(\Pi_{a} \otimes \Pi_{F}\right)(\mathscr{A} \otimes \mathscr{W}(E))^{\prime \prime}=\mathscr{M}_{a} \bar{\otimes} \mathscr{B}\left(F_{+}\right)
$$

(remember $\Pi_{F}(\mathscr{W}(E))^{\prime \prime}=\mathscr{B}\left(F_{+}\right)[14$, Proposition 5.2.4]). By duality

$$
\left\langle v_{t}(\omega) ; Z\right\rangle=\left\langle\omega ; \mathrm{e}^{i t H} Z \mathrm{e}^{-i t H}\right\rangle \quad \forall Z \in \mathscr{M}_{a, F} \quad \forall \omega \in \mathscr{F}_{a, F} \quad \forall t \in \boldsymbol{R}
$$

the limiting dynamics is obtained in the Schrödinger picture as a group $\left(v_{t}\right)_{t \in \boldsymbol{R}}$ of affine, bijective maps on $\mathscr{F}_{a . F}$ with $\lim _{t \rightarrow 0}\left\|v_{t}(\omega)-\omega\right\|=0 \quad \forall \omega \in \mathscr{F}_{a, F}$. Obviously, if $\varrho_{\omega}$ is any density operator on $\mathscr{H}_{a, F}$ representing $\omega \in \mathscr{F}_{a, F}$,

$$
\langle\omega ; Z\rangle=\operatorname{tr}\left[\varrho_{\omega} Z\right] \quad \forall Z \in \mathscr{M}_{a, F},
$$

the Schrödinger dynamics is given in the usual form $v_{t}(\omega) \cong \mathrm{e}^{-i t H} \varrho_{\omega} \mathrm{e}^{i t H}$.

In the present paper we investigate the time evolution of the electromagnetic field, that is, we are interested in the restrictions $\left.v_{t}(\omega)\right|_{b}$ of the time evolved states $v_{t}(\omega), t \in \boldsymbol{R}$, to the bosonic part (photons):

$$
\left\langle\left. v_{t}(\omega)\right|_{b} ; Y\right\rangle=\left\langle v_{t}(\omega) ; 1_{\mathscr{A}} \otimes Y\right\rangle \quad \forall Y \in \mathscr{W}(E)
$$

Clearly, $\left.v_{t}(\omega)\right|_{b}$ defines for each $t \in \boldsymbol{R}$ and every $\omega \in \mathscr{F}_{a, \boldsymbol{F}}$ a state on the photon $C^{*}$-algebra $\mathscr{W}(E)$, which is normal to the Fock representation.

Let us at this point introduce some notations concerning states on the Weyl algebra $\mathscr{W}(E)$ (see also [1, Section 3]).

Because of the Weyl commutation relations [14, Theorem 5.2.8] each state $\varphi$ on $\mathscr{W}(E)$ is uniquely determined by its characteristic function

$$
C_{\varphi}: E \rightarrow C, f \mapsto\langle\varphi ; W(f)\rangle=C_{\varphi}(f),
$$

the expectation values with the Weyl operators $W(f) \in \mathscr{W}(E), f \in E$, [8].

For a regular state $\varphi$ on $\mathscr{W}(E)$ we denote the field, creation, resp. annihilation operators associated with the GNS-representation $\left(\Pi_{\varphi}, \mathscr{H}_{\varphi}, \Omega_{\varphi}\right)$ of $\varphi$ by $\Phi_{\Pi_{\varphi}}(f)=\Phi_{\varphi}(f), a_{\Pi_{\varphi}}^{*}(f)=a_{\varphi}^{*}(f)$, resp. $a_{\Pi_{\varphi}}(f)=a_{\varphi}(f), f \in E$.

The Fock representation $\Pi_{F}$ is just the GNS-representation of the Fock 
state $\omega_{F}$ on $\mathscr{W}(E)$ with associated characteristic function

$$
C_{F}(f)=\left\langle\omega_{F} ; W(f)\right\rangle=\exp \left\{-\frac{1}{4}\|f\|^{2}\right\} \quad \forall f \in E,
$$

and the Fock vacuum vector $\Omega_{F}$ as cyclic vector. The Weyl operators $W_{F}(f)$ acting on the Fock space $F_{+}$are connected with the abstract $W(f) \in \mathscr{W}(E)$ by the representation morphism $\Pi_{F}(W(f))=W_{F}(f), f \in E$.

In the case of an analytic state $\varphi$ on $\mathscr{W}(E)$ the cyclic vector $\Omega_{\varphi}$ is contained in the domain of every polynomial of field operators $\Phi_{\varphi}(f)$, because of which one commonly defines the expectation values of the unbounded field expressions as

$$
\left\langle\varphi ; \Phi_{\varphi}\left(f_{1}\right) \cdots \Phi_{\varphi}\left(f_{m}\right)\right\rangle:=\left\langle\Omega_{\varphi} \mid \Phi_{\varphi}\left(f_{1}\right) \cdots \Phi_{\varphi}\left(f_{m}\right) \Omega_{\varphi}\right\rangle
$$

for arbitrary $f_{1}, \cdots, f_{m} \in E$ and $m \in \mathbb{N}[14]$.

\section{§3. The Photon Field at Large Times}

The existence of the time asymptotic states $R_{t}(\omega), t \in \mathbb{R}, \omega \in \mathscr{F}_{a, F}$, on $\mathscr{W}(E)$ in the sense of $(1.1)$ is based essentially on two facts. First, since the one-photon Hamiltonian $\sqrt{-\Delta}$ has a purely absolutely continuous spectrum, one obtains

$$
\lim _{t \rightarrow \infty}\langle f \mid \exp \{i t \sqrt{-\Delta}\} g\rangle=0 \quad \forall f, g \in \mathbb{L}^{2}\left(\boldsymbol{R}^{3}\right)
$$

a result from scattering theory, [15] p. 110ff. Secondly, we have to perform the infinite time limit of the (bounded) linear forms $f \in E \mapsto\left\langle h_{t} \mid f\right\rangle, t \in \mathbb{R}$, where

$$
h_{t}:=-i \int_{0}^{t} \exp \{-i \tau(\sqrt{-\Delta}-\varepsilon)\} \phi \mathrm{d} \tau \in \mathrm{L}^{2}\left(\mathbb{R}^{3}\right)
$$

with the coupling function $\phi$ of (2.5), and $\varepsilon>0$, the level-splitting of a single atom. To obtain the existence of some limiting linear form $L: E \rightarrow C$ with

$$
L(f)=\lim _{t \rightarrow \infty}\left\langle h_{t} \mid f\right\rangle \quad \forall f \in E
$$


we however have to specialize the one-photon testfunction space $E$. For this we introduce in momentum space $\mathbb{R}^{3}$ the energy sphere of resonant modes

$$
S_{\varepsilon}:=\left\{k \in \mathbb{R}^{3}|| k \mid=\varepsilon\right\} .
$$

If $F$ denotes the (unitary) Fourier transformation on $\mathrm{L}^{2}\left(\boldsymbol{R}^{3}\right)^{1}$,

$$
\left(\boldsymbol{F}(f)(k)=\hat{f}(k)=(2 \pi)^{-3 / 2} \int_{\boldsymbol{R}^{3}} \mathrm{e}^{-i k \cdot x} f(x) \mathrm{d}^{3} x, \quad f \in \mathrm{L}^{2}\left(\boldsymbol{R}^{3}\right),\right.
$$

we demand $E$ to be a subspace of

$$
E_{\max }:=\left\{f \in \mathrm{L}^{2}\left(\mathbb{R}^{3}\right) \mid \hat{f} \text { is } \mathscr{C}^{1} \text { in a neighborhood of } S_{\varepsilon}\right\},
$$

where $\mathscr{C}^{k}$ for $k \in \mathbb{N} \cup\{\infty\}$ means the $k$-times continuously differentiable functions. According to Section $2 E$ also has to fulfill (2.1) and to be dense in $\mathrm{L}^{2}\left(\mathbb{R}^{3}\right)$.

For the existence of the limits in (3.3) we also have to assume the coupling function $\phi$ to be an element of $E_{\max }$.

Proposition 3.1. Let $E$ be an arbitrary subspace of $E_{\max }$ and $\phi \in E_{\max }$. Then the limits (3.3) exist, and the limiting linear form $L: E \rightarrow C$ is given as the sum

$$
L(f)=L^{\prime}(f)+L^{\prime \prime}(f) \quad \forall f \in E
$$

with the linear forms $L^{\prime}$ and $L^{\prime \prime}$

$$
\begin{aligned}
& L^{\prime}(f)=i \pi \int_{S_{\varepsilon}} \bar{\phi}(k) \hat{f}(k) \mathrm{d} S(k), \\
& L^{\prime \prime}(f)=-\mathrm{pv} \int_{\mathbb{R}^{3}} \frac{\bar{\phi}(k) \hat{f}(k)}{|k|-\varepsilon} \mathrm{d}^{3} k,
\end{aligned}
$$

where $\mathrm{d} S(k)$ is the surface measure of the sphere $S_{\varepsilon}$ and $\mathrm{pv}$ denotes the principle value.

${ }^{1}$ It is $a \cdot b:=\sum_{k=1}^{3} a_{k} b_{k}$ for all $a=\left(a_{1}, a_{2}, a_{3}\right), b=\left(b_{1}, b_{2}, b_{3}\right) \in C^{3}$. 
Proof. From the theory of distributions it is well known (observe, $(t, x) \mapsto$ $\int_{0}^{t} \mathrm{e}^{i \tau x} \mathrm{~d} \tau=\frac{\mathrm{e}^{i t x}-1}{i x}$ is continuous) [16] that

$$
\lim _{t \rightarrow \infty} \int_{\mathbb{R}} \psi(y) \int_{0}^{t} \exp \{i \tau(y-\varepsilon)\} \mathrm{d} \tau \mathrm{d} y=\pi \psi(\varepsilon)+i \mathrm{pv} \int_{\mathbb{R}} \frac{\psi(y)}{y-\varepsilon} \mathrm{d} y
$$

which can be generalized to functions $\psi \in \mathrm{L}^{1}(\boldsymbol{R})$ which are $\mathscr{C}^{1}$ in a neighborhood of $\varepsilon \in(\boldsymbol{R})$. Consequently, by splitting the integral into its radial component and a spherical integral (cf. e.g. [17] §14 Satz 8), equation (3.9) leads for those $h \in \mathrm{L}^{1}\left(\mathbb{R}^{3}\right)$ which are $\mathscr{C}^{1}$ in a neighborhood of $S_{\varepsilon}, \varepsilon>0$, to

$$
\lim _{t \rightarrow \infty} \int_{\mathbb{R}^{3}} h(k) \int_{0}^{t} \exp \{i \tau(|k|-\varepsilon)\} \mathrm{d} \tau \mathrm{d}^{3} k=\pi \int_{S_{\varepsilon}} h(k) \mathrm{d} S(k)+i \mathrm{pv} \int_{\mathbb{R}^{3}} \frac{h(k)}{|k|-\varepsilon} \mathrm{d}^{3} k,
$$

where the principal value is taken with respect to the radial integral. Now, using the Fourier transformation $\mathbb{F}$ with (3.2) we obtain

$$
\begin{aligned}
\left\langle h_{t} \mid f\right\rangle & =i \int_{0}^{t}\left\langle\mathrm{e}^{-i \tau(\sqrt{-\Delta}-\varepsilon)} \phi \mid f\right\rangle \mathrm{d} \tau=i \int_{0}^{t}\left\langle F \phi \mid \mathrm{e}^{i \tau(|\cdot|-\varepsilon)} \mathbb{F} f\right\rangle \mathrm{d} \tau \\
& =i \int_{\mathbb{R}^{3}} \overline{\phi(k)} \hat{f}(k) \int_{0}^{t} \mathrm{e}^{i \tau(|k|-\varepsilon)} \mathrm{d} \tau \mathrm{d}^{3} k .
\end{aligned}
$$

For $\phi, f \in E_{\max }$ the function $k \in \mathbb{R}^{3} \mapsto \bar{\phi}(k) \hat{f}(k)$ is $\mathrm{L}^{1}$ on $\mathbb{R}^{3}$ and $\mathscr{C}^{1}$ in a neighborhood of $S_{\varepsilon}$.

Before proceeding let us give some examples for the testfunction space $E$. Observe that $F \exp \{i t \sqrt{-\Delta}\} F^{-1}$ is the multiplication operator with the continuous function $k \in \mathbb{R}^{3} \mapsto \exp \{i t|k|\}$, which is $\mathscr{C}^{\infty}$ except at the origin $k=0$. Define $\mathscr{C}_{c, 0}^{\infty}\left(\mathbb{R}^{3}\right)$ to be the space of all complex-valued $\mathscr{C}^{\infty}$-functions on $\boldsymbol{R}^{3}$ with compact support and vanishing in a neighborhood of the origin. Further, write $f \in \mathscr{C}_{c, 0, \varepsilon}^{\infty}\left(\mathbb{R}^{3}\right)$, if and only if $f \in \mathscr{C}_{c, 0}^{\infty}\left(\mathbb{R}^{3}\right)$ and $f$ vanishes in a neighborhood of the sphere $S_{\varepsilon}$. Some appropriate choices for $\mathbb{E}$ are given by

$$
E_{\max } ; F^{-1}\left(\mathscr{C}\left(\mathbb{R}^{3}\right) \cap E_{\max }\right) ; \mathbb{F}^{-1}\left(\mathscr{C}_{c, 0}^{\infty}\left(\mathbb{R}^{3}\right)\right) ; \mathbb{F}^{-1}\left(\mathscr{C}_{c, 0, \varepsilon}^{\infty}\left(\mathbb{R}^{3}\right)\right)
$$


each of them fulfilling the above conditions. $\left(\mathscr{C}\left(\boldsymbol{R}^{3}\right)\right.$ are the continuous functions on $R^{3}$.) In the following we choose $E$ always to satisfy

$$
F^{-1}\left(\mathscr{C}_{c, 0, \varepsilon}^{\infty}\left(\mathbb{R}^{3}\right)\right) \subseteq E \subseteq E_{\max }
$$

After this preliminary discussion we now turn to the time asymptotic states $R_{t}(\omega)$ of (1.1). With (2.4) and (2.11) one has

$$
\mathscr{M}_{a, F}=\mathscr{M}_{0} \bar{\otimes} \mathrm{L}^{\infty}\left(T_{\eta, \gamma}, \lambda^{\eta, \gamma}\right) \bar{\otimes} \mathscr{B}\left(F_{+}\right)
$$

The restriction of $\omega \in \mathscr{F}_{a, F}$ to $\mathrm{L}^{\infty}\left(T_{\eta, \gamma}, \lambda^{\eta, \gamma}\right)$-more exactly, to $\mathbb{1}_{0} \otimes \mathrm{L}^{\infty}\left(T_{\eta, \gamma}, \lambda^{\eta, \gamma}\right)$ $\otimes \mathbb{1}_{F}$-leads to a probability measure $\rho_{\omega} \in M_{+}^{1}\left(T_{\eta, \gamma}\right)$ absolutely continuous to $\lambda^{\eta, \gamma}$ :

$$
\rho_{\omega}(G)=\int_{T_{\eta, \gamma}} G(\vartheta) \mathrm{d} \rho_{\omega}(\vartheta)=\left\langle\omega ; \mathbb{1}_{0} \otimes G \otimes \mathbb{1}_{F}\right\rangle=\operatorname{tr}\left[\varrho_{\omega}\left(\mathbb{1}_{0} \otimes G \otimes \mathbb{1}_{F}\right)\right]
$$

for each $G \in \mathrm{L}^{\infty}\left(T_{\eta, \gamma}, \lambda^{\eta \cdot \gamma}\right)$ and with a density operator $\varrho_{\omega}$ satisfying (2.13). Since each $\psi \in \mathscr{H}_{a, F} \cong \int_{T_{\eta, \gamma}}^{\oplus} \mathscr{H}_{0} \otimes F_{+} \mathrm{d} \lambda^{\eta, \gamma}(\vartheta)$ is isomorphic to a square integrable, $\mathscr{H}_{0} \otimes F_{+}$-valued function we find the following result concerning the measure $\rho_{\omega} \in M_{+}^{1}\left(T_{\eta, \gamma}\right)$.

Lemma 3.2. Let $2_{w}=\sum_{n=1}^{N} a_{n}\left|\psi_{n}\right\rangle\left\langle\psi_{n}\right|, a_{n}>0$, with some $N \in N \cup\{\infty\}$ be the spectral decomposition of a density operator $\varrho_{\omega}$ on $\mathscr{H}_{a, F}$ associated with $\omega \in \mathscr{F}_{a, F}$ via (2.13). Then with $\psi_{n} \cong \int_{T_{\eta, \gamma}}^{\oplus} \psi_{n}(\vartheta) \mathrm{d} \lambda^{\eta, \gamma}($,$) the function \vartheta \in T_{\eta, \gamma} \mapsto$ $\left.\sum_{n=1}^{N} a_{n} \| \psi_{n}(),\right) \|^{2}$ is an element of $\mathrm{L}^{1}\left(T_{\eta, \gamma}, \lambda^{\eta, \gamma}\right)$, and the measure $\rho_{\omega} \in M_{+}^{1}\left(T_{\eta, \gamma}\right)$ has the form

$$
\mathrm{d} \rho_{\omega}(\ell)=\left(\sum_{n=1}^{N} a_{n}\left\|\psi_{n}(,)\right\|^{2}\right) \mathrm{d} \lambda^{\eta, \gamma}(\ell)
$$

Proof. $\quad Q \mapsto \sum_{n=1}^{N} a_{n}\left\|\psi_{n}(\vartheta)\right\|^{2}$ being an element of $\mathrm{L}^{1}\left(T_{\eta, \gamma}, \lambda^{\eta, \gamma}\right)$ is an im- 
mediate consequence of the theory of direct integrals. Since $\mathbb{H}_{0} \otimes G \otimes \mathbb{H}_{F} \cong$ $\int_{T_{\eta, \gamma}}^{\oplus} G(\vartheta) \mathbb{1}_{0} \otimes \mathbb{1}_{F} \mathrm{~d} \lambda^{\eta, \gamma}(\ell)$ one easily checks

$$
\begin{aligned}
\operatorname{tr}\left[\varrho_{\omega}\left(\mathbb{1}_{0} \otimes G \otimes \mathbb{1}_{F}\right)\right] & =\sum_{n=1}^{N} a_{n}\left\langle\psi_{n} \mid\left(\mathbb{1}_{0} \otimes G \otimes \mathbb{1}_{F}\right) \psi_{n}\right\rangle \\
& =\sum_{n=1}^{N} a_{n} \int_{T_{n, \nu}} G(\vartheta)\left\|\psi_{n}(\vartheta)\right\|^{2} \mathrm{~d} \lambda^{\eta, \gamma}(\vartheta),
\end{aligned}
$$

from which the result follows by observing that $G$ is uniformly bounded and by use of Lebesgue's dominated convergence theorem.

Lemma 3.2 also shows that the measure $\rho_{\omega}$ is absolutely continuous with respect to $\lambda^{\eta, \gamma}$.

We now arrive at the main theorem of the present section, which ensures the existence of the time asymptotic states $R_{t}(\omega)$ on the Weyl algebra $\mathscr{W}(E)$.

Theorem 3.3. The following two assertions are valid:

(a) For each $\omega \in \mathscr{F}_{a, F}$ and every $t \in \mathbb{R}$ the characteristic function

$$
\left\langle R_{t}(\omega) ; W(f)\right\rangle:=C_{F}(f) \int_{[0,2 \pi[} \exp \left\{i \sqrt{2} \beta \lambda \operatorname{Re}\left(\mathrm{e}^{i \vartheta} L(f)\right)\right\} \mathrm{d}\left(\rho_{\omega^{\circ}} \varphi_{-t}^{\eta, \gamma}(\vartheta)\right)
$$

defines a state $R_{t}(\omega)$ on $\mathscr{W}(E)$.

(b) With the states $R_{t}(\omega)$ of (a) we have the time asymptotic relations (1.1):

$$
\underset{t \rightarrow \infty}{\text { weak}^{*}-\lim }\left(\left.v_{t}(\omega)\right|_{b}-R_{t}(\omega)\right)=0
$$

for each $\omega \in \mathscr{F}_{a, F}$.

Proof. (a) Since $\omega_{F}$ is a state on $\mathscr{W}(E)$, its characteristic function $C_{F}$ constitutes the positive definite kernel $(f, g) \in E \times E \mapsto \exp \left\{\frac{i}{2} \operatorname{Im}\langle f \mid g\rangle\right\} C_{F}(g-f)$ 
on $E \times E$. Because the map $f \in E \mapsto\left\langle R_{t}(\omega) ; W(f)\right\rangle=: C(f)$ is the product of $C_{F}$ and a positive definite function on $E$, the kernel $(f, g) \in E \times E \mapsto$ $\exp \left\{\frac{i}{2} \operatorname{Im}\langle f \mid g\rangle\right\} C(g-f)$ is also positive definite and hence defines a state on $\mathscr{W}(E)([8]$, Appendix).

(b) For the proof of (b) we need several steps.

(i) Using (2.10) for each $f \in \mathrm{L}^{2}\left(\boldsymbol{R}^{3}\right)$ with the substitution $\tau$ : $=t-u$ we get

$$
\begin{aligned}
\operatorname{Im}\left\langle f(t, \vartheta) \mid \mathrm{e}^{i t \sqrt{-\Delta}} f\right\rangle & =\sqrt{2} \beta \lambda \operatorname{Im}\left(\mathrm{e}^{i(\vartheta+\varepsilon t)} \int_{u=0}^{t}\left\langle\mathrm{e}^{i(u-t)(\sqrt{-\Delta}-\varepsilon)} \phi \mid f\right\rangle \mathrm{d} u\right) \\
& =\sqrt{2} \beta \lambda \operatorname{Im}\left(\mathrm{e}^{i(\vartheta+\varepsilon t)} \int_{u=0}^{t}\left\langle\mathrm{e}^{-i \tau \sqrt{-\Delta}-\varepsilon)} \phi \mid f\right\rangle \mathrm{d} \tau\right) \\
& \stackrel{(2.6)}{=}-\sqrt{2} \beta \lambda \operatorname{Re}\left(\mathrm{e}^{i \varphi_{t}^{\eta, \gamma}(\vartheta)}\left\langle h_{t} \mid f\right\rangle\right)
\end{aligned}
$$

with $h_{t}$ from (3.2) and the flow $\varphi_{t}^{\eta, \gamma}$ on $T_{\eta, \gamma}$ from (2.6).

(ii) With (2.14) and (3.1) one immediately checks

$$
\lim _{t \rightarrow \infty} C_{F}(\exp \{i t \sqrt{-\Delta}\} f+g)=C_{F}(f) C_{F}(g) \quad \forall f, g \in E
$$

(iii) Define for each $t \in R$ and $f \in E$ the following functions on $T_{\eta, \gamma}$

$$
F_{t}^{f}(\vartheta):=\exp \left\{i \sqrt{2} \beta \lambda \operatorname{Re}\left(\mathrm{e}^{i \vartheta}\left\langle h_{t} \mid f\right\rangle\right)\right\}, \quad F^{f}(\vartheta):=\exp \left\{i \sqrt{2} \beta \lambda \operatorname{Re}\left(\mathrm{e}^{i \vartheta} L(f)\right)\right\}
$$

Obviously $\lim \left\|F_{t}^{f}-F^{f}\right\|_{\infty}=0$ by (3.3) resp. Proposition 3.1 .

$$
t \rightarrow \infty
$$

(iv) Now choose vectors $\theta_{k}=\xi_{k} \otimes W_{F}\left(h_{k}\right) \Omega_{F}, k=1,2$, where $\xi_{k} \in \mathscr{H}_{a}$ (cf. eq. (2.3)) and $h_{k} \in E ; \Omega_{F}$ is the Fock vacuum vector. For $f \in E$ we obtain with (2.9), the Weyl relation, and the equations (3.12) and (2.7)

$$
\begin{aligned}
& \left\langle\theta_{1} \mid \mathrm{e}^{i t H}\left(1_{a} \otimes W_{F}(f)\right) \mathrm{e}^{-i t H} \theta_{2}\right\rangle= \\
& \quad \stackrel{(3.12)}{=}\left\langle\xi_{1} \mid\left(\mathbb{1}_{0} \otimes\left(F_{t}^{f} \circ \varphi_{t}^{\eta, \gamma}\right)\right) \xi_{2}\right\rangle\left\langle\omega_{F} ; W\left(-h_{1}\right) W\left(\mathrm{e}^{i t \sqrt{-\Delta}} f\right) W\left(h_{2}\right)\right\rangle
\end{aligned}
$$




$$
\begin{aligned}
\stackrel{(2.7)}{=} & \left\langle\mathrm{e}^{-i t A} \xi_{1} \mid\left(\mathbb{1}_{0} \otimes F_{t}^{f}\right) \mathrm{e}^{-i t A} \xi_{2}\right\rangle \\
& \times \exp \left\{\frac{i}{2}\left[\operatorname{Im}\left\langle h_{1} \mid h_{2}\right\rangle+\operatorname{Im}\left\langle h_{1}+h_{2} \mid \mathrm{e}^{i t \sqrt{-\Delta}} f\right\rangle\right]\right\} C_{F}\left(\mathrm{e}^{i t \sqrt{-\Delta}} f+\left(h_{2}-h_{1}\right)\right) .
\end{aligned}
$$

Obviously $\left|\left\langle\mathrm{e}^{-i t A} \xi_{1} \mid\left(\mathbb{1}_{0} \otimes\left(F_{t}^{f}-F^{f}\right)\right) \quad \mathrm{e}^{-i t A} \xi_{2}\right\rangle\right| \leq\left\|\xi_{1}\right\|\left\|\xi_{2}\right\|\left\|F_{t}^{f}-F^{f}\right\|_{\infty} \stackrel{t \rightarrow \infty}{\longrightarrow} 0$, from which together with (3.13) and the observation

$$
\exp \left\{\frac{i}{2} \operatorname{Im}\left\langle h_{1} \mid h_{2}\right\rangle\right\} C_{F}\left(h_{2}-h_{1}\right)=\left\langle W_{F}\left(h_{1}\right) \Omega_{F} \mid W_{F}\left(h_{2}\right) \Omega_{F}\right\rangle
$$

follows

$$
\begin{aligned}
\lim _{t \rightarrow \infty} \mid\left\langle\theta_{1} \mid \mathrm{e}^{i t H}\left(\mathbb{1}_{a} \otimes W_{F}(f)\right) \mathrm{e}^{-i t H} \theta_{2}\right\rangle & \\
& \quad-C_{F}(f)\left\langle\theta_{1} \mid\left(\mathbb{1}_{0} \otimes\left(F^{f} \circ \varphi_{t}^{\eta, \gamma}\right) \otimes \mathbb{1}_{F}\right) \theta_{2}\right\rangle \mid=0 .
\end{aligned}
$$

(v) From the fact that $\left\{\xi \otimes W_{F}(h) \Omega_{F} \mid \xi \in \mathscr{H}_{a}, h \in E\right\}$ is total in $\mathscr{H}_{a, F}=$ $\mathscr{H}_{a} \otimes F_{+}$(cf. eq. (2.3)) by an $\frac{\varepsilon}{3}$-argument the validity of (3.15) for all $\theta_{1}, \theta_{2} \in \mathscr{H}_{a, F}$ follows. With (2.12), (2.13) and (3.11) we finally obtain the assertion from the substitution $\int F^{f} \circ \varphi_{t}^{\eta, \gamma}(\vartheta) \mathrm{d} \rho_{\omega}(\vartheta)=\int F^{f}(\vartheta) \mathrm{d}\left(\rho_{\omega} \circ \varphi_{-t}^{\eta, \gamma}(),\right)$.

For special product states $\omega \in \mathscr{F}_{a, F}$ one obtains by direct calculation an explicit closed expression for the characteristic functions associated with the states $\left.v_{t}(\omega)\right|_{b}$. If $\mu$ is a probability measure on $\mathrm{L}^{2}\left(\boldsymbol{R}^{3}\right), \mu \in M_{+}^{1}\left(\mathrm{~L}^{2}\left(\mathbb{R}^{3}\right)\right)$, then by $\left(\Omega_{F}\right.$ is the Fock vacuum vector)

$$
\int_{L^{2}\left(R^{3}\right)}\left|W_{F}(h) \Omega_{F}\right\rangle\left\langle W_{F}(h) \Omega_{F}\right| \mathrm{d} \mu(h)
$$

there is defined a density operator on the Fock space $F_{+}$. The $W_{F}(h) \Omega_{F}$, $h \in \mathrm{L}^{2}\left(\boldsymbol{R}^{3}\right)$ are the so-called Glauber vectors, which are total in $F_{+}$. Expression (3.16) is a statistical mixture of pure Glauber vector states, the so-called $P$-representation (cf. [12] and the section on classical states in 
[18]). The Fock-normal state on $\mathscr{W}(E)$ associated with the density operator (3.16) are denoted by $\omega_{F}^{\mu}$. With the Weyl relations one immediately checks

$$
\left\langle\omega_{F}^{\mu} ; W(f)\right\rangle=C_{F}(f) \int_{\mathbf{L}^{2}\left(R^{3}\right)} \exp \{i \operatorname{Im}\langle h \mid f\rangle\} \mathrm{d} \mu(h) \quad \forall f \in E
$$

Obviously, if $\mu=\delta_{0}$ is the point measure at zero, then we have $\omega_{F}^{\delta_{0}}=\omega_{F}$, the Fock state $(2.14)$ on $\mathscr{W}(E)$. For an arbitrary $\omega_{a} \in \mathscr{F}_{a}$ the product state $\omega_{a} \otimes \omega_{F}^{\mu}$ is an element of the folium $\mathscr{F}_{a, F}$

Proposition 3.4. For $\mu \in M_{+}^{1}\left(\mathrm{~L}^{2}\left(R^{3}\right)\right)$ and $\omega_{a} \in \mathscr{F}_{a}$ one has for each $f \in E$ and $t \in \boldsymbol{R}$

$$
\begin{aligned}
& \left\langle v_{t}\left(\omega_{a} \otimes \omega_{F}^{\mu}\right) ; W(f)\right\rangle \\
& =C_{F}(f) \int_{[0,2 \pi[} \exp \left\{i \sqrt{2} \beta \lambda \operatorname{Re}\left(\mathrm{e}^{i \vartheta}\left\langle h_{t} \mid f\right\rangle\right)\right\} \mathrm{d}\left(\tilde{\rho}_{\omega_{a}} \circ \varphi_{-t}^{\eta, \gamma}(\vartheta)\right) \\
& \quad \times \int_{L^{2}\left(R^{3}\right)} \exp \left\{i \operatorname{Im}\left\langle h \mid \mathrm{e}^{i t \sqrt{-\Delta}} f\right\rangle\right\} \mathrm{d} \mu(h),
\end{aligned}
$$

where $\tilde{\rho}_{\omega_{a}} \in M_{+}^{1}\left(T_{\eta, \gamma}\right)$ is obtained by the restriction of $\omega_{a}$ to $\mathrm{L}^{\infty}\left(T_{\eta, \gamma}, \lambda^{\eta, \gamma}\right)$, similar to (3.11): $\tilde{\rho}_{\omega_{a}}=\rho_{\omega_{a} \otimes \omega_{F}^{\mu}}$.

Proof. Analogously to (3.14) one gets with $C_{F}\left(\mathrm{e}^{i t \sqrt{-\Delta}} f\right)=C_{F}(f) \forall f \in E$

$$
\begin{aligned}
& \quad\left\langle\omega_{a} \otimes \omega_{F}^{\mu} ; \mathrm{e}^{i t H}\left(1_{a} \otimes W_{F}(f)\right) \mathrm{e}^{-i t H}\right\rangle \\
& =\left\langle\omega_{a} ; \mathbf{1}_{0} \otimes\left(F_{t}^{f} \circ \varphi_{t}^{\eta, \gamma}\right)\right\rangle\left\langle\omega_{F}^{\mu} ; W\left(\mathrm{e}^{i t \sqrt{-\Delta}} f\right)\right\rangle \\
& \stackrel{(3.17)}{=} \int_{[0,2 \pi[} F_{t}^{f} \circ \varphi_{t}^{\eta, \gamma}(\vartheta) \mathrm{d} \tilde{\rho}_{\omega_{a}}(\vartheta) C_{F}(f) \int_{L^{2}\left(R^{3}\right)} \exp \left\{i \operatorname{Im}\left\langle h \mid \mathrm{e}^{i t \sqrt{-\Delta}} f\right\rangle\right\} \mathrm{d} \mu(h) .
\end{aligned}
$$

From formula (3.18) one recognizes how the time asymptotic states $R_{t}\left(\omega_{a} \otimes \omega_{F}^{\mu}\right)$ given by

$$
\left\langle R_{t}\left(\omega_{a} \otimes \omega_{F}^{\mu}\right) ; W(f)\right\rangle=C_{F}(f) \int_{[0,2 \pi[} \exp \left\{i \sqrt{2} \beta \lambda \operatorname{Re}\left(\mathrm{e}^{i, \vartheta} L(f)\right)\right\} \mathrm{d}\left(\tilde{\rho}_{\omega_{a}} \circ \varphi_{t}^{\eta, \gamma}(\vartheta)\right)
$$


emerge from the full Schrödinger dynamically evolved states $v_{t}\left(\omega_{a} \otimes \omega_{F}^{\mu}\right)$ by means of the limits (3.1) and (3.3).

Since the flow $\varphi_{t}^{\eta, \gamma}$ is a rotation on $T_{\eta, \gamma}$ with period $\frac{2 \pi}{\varepsilon}$ (cf. eq. (2.6)) it seems reasonable to average over the period, or to perform the more general limit by means of the Cesàro averages

$$
\operatorname{Lim}_{t \rightarrow \infty} k(t):=\lim _{t \rightarrow \infty} t^{-1} \int_{0}^{t} k(\tau) \mathrm{d} \tau
$$

noting that if the ordinary $\operatorname{limit} \lim k(t)=a$ exists, so does the generalized $t \rightarrow \infty$

$\operatorname{Lim} k(t)=a([15$, Lemma 4.18], cf. also [6]). $t \rightarrow \infty$

Corollary 3.5. The following two assertions are valid:

(a) For each $\omega \in \mathscr{F}_{a, F}$ the characteristic function

$$
\begin{aligned}
\left\langle\bar{R}_{\eta, \gamma} ; W(f)\right\rangle:= & C_{F}(f) \int_{[0,2 \pi[} \exp \left\{i \sqrt{2} \beta \lambda \operatorname{Re}\left(\mathrm{e}^{i, \varphi} L(f)\right)\right\} \mathrm{d} \lambda^{\eta, \gamma}(\vartheta) \\
& =C_{F}(f) J_{0}(\beta \lambda|L(f)|)
\end{aligned}
$$

defines a state $\bar{R}_{\eta, \gamma}$ on $\mathscr{W}(E)$, where $J_{0}$ denotes the zero order Bessel function.

(b) It holds for each $s \in \boldsymbol{R}$ and every $\omega \in \mathscr{F}_{a, F}$

$$
\begin{aligned}
\underset{t \rightarrow \infty}{\text { weak }_{t \rightarrow \infty}-\left.\operatorname{Lim} v_{t}(\omega)\right|_{b}} & =\left.\underset{t \rightarrow \infty}{\text { weak }_{t \rightarrow \infty}^{*}-\lim } \frac{\varepsilon}{2 \pi} \int_{t}^{t+2 \pi / \varepsilon} v_{\tau}(\omega)\right|_{b} \mathrm{~d} \tau \\
& =\frac{\varepsilon}{2 \pi} \int_{s}^{s+2 \pi / \varepsilon} R_{\tau}(\omega) \mathrm{d} \tau=\bar{R}_{\eta, \gamma} .
\end{aligned}
$$

Proof. Observe that $\Delta \in \mathscr{B}\left(T_{\eta, \gamma}\right) \mapsto \frac{\varepsilon}{2 \pi} \int_{s}^{s+2 \pi / \varepsilon} \rho_{\omega}\left(\varphi_{-\tau}^{\eta, \gamma}(\Delta)\right) \mathrm{d} \tau$ is just the normalized Lebesgue measure $\mathrm{d} \lambda^{\eta, \gamma}()=,\frac{\mathrm{d} \ell}{2 \pi}$ on $T_{\eta, \gamma}=\left[0,2 \pi\left[\left(\mathscr{B}\left(T_{\eta, \gamma}\right)\right.\right.\right.$ are the Borel subsets of $\left.T_{\eta, \gamma}\right)$. The remaining argumentation is obvious. 
Clearly, the measure $\rho_{\omega}$ is invariant under the flow $\left(\varphi_{t}^{\eta, \gamma}\right)_{t \in \mathbb{R}}$, if and only if $\rho_{\omega}=\lambda^{\eta, \gamma}$. In this case $R_{t}(\omega)=\bar{R}_{\eta, \gamma} \forall t \in R$, and for such states $\omega \in \mathscr{F}_{a . F}$ the usual limit exits according to

$$
\left.\underset{t \rightarrow \infty}{\operatorname{weak}}{ }_{t \rightarrow-\lim } v_{t}(\omega)\right|_{b}=\bar{R}_{\eta, \gamma^{*}}
$$

\section{$\S 4$. Properties of the Emitted Radiation}

For an initial $\Pi_{a} \otimes \Pi_{F}$-normal state $\omega \in \mathscr{F}_{a, F}$ on $\mathscr{A} \otimes \mathscr{W}(E)$ the time evolution is given by the Schrödinger dynamics $\left(v_{t}\right)_{t \in \mathbb{R}}$, i.e. by the map $t \in \mathbb{R} \mapsto v_{t}(\omega)$ (cf. formula (2.12)). Its restriction $\left.t \in \boldsymbol{R} \mapsto v_{t}(\omega)\right|_{b}$ to the photon system describes the quantum states of the evolving radiation field, which are obtained by emission and absorption processes up to the time $t>0$. However for large times $t>>$ the time asymptotic states $R_{t}(\omega)$, resp. the averaged one $\bar{R}_{\eta, \gamma}$ of Corollary 3.5, are appropriate for the description of the electromagnetic field by Theorem 3.3. Denote by

$$
\mathscr{R}_{\mathrm{as}}:=\left\{R_{t}(\omega) \mid t \in \boldsymbol{R}, \omega \in \mathscr{F}_{a, F}\right\} \ni \bar{R}_{\eta, \gamma}
$$

the set of all time asymptotic states on $\mathscr{W}(E)$. Obviously, there is a one-to-one correspondence between $\mathscr{R}_{\text {as }}$ and the measures on $[0,2 \pi$ [, which are absolutely continuous to $\lambda^{\eta, \gamma}$, that is, between $\mathscr{R}_{a s}$ and $\mathrm{L}^{1}\left(\left[0,2 \pi\left[, \lambda^{\eta, \gamma}\right)\right.\right.$.

\subsection{Quantum optical coherence}

Theorem 4.1. Let $\varphi \in \mathscr{R}_{\text {as }}$. Then $\varphi$ is entire-analytic, and with the linear form $L: E \rightarrow C$ of Proposition 3.1 we have the factorization

$$
\left\langle\varphi ; a_{\varphi}^{*}\left(f_{1}\right) \cdots a_{\varphi}^{*}\left(f_{m}\right) a_{\varphi}\left(g_{1}\right) \cdots a_{\varphi}\left(g_{m}\right)\right\rangle=(\beta \lambda)^{2 m} L\left(f_{1}\right) \cdots L\left(f_{m}\right) \overline{L\left(g_{1}\right)} \cdots \overline{L\left(g_{m}\right)}
$$

for all $f_{1}, \cdots, f_{m}, g_{1}, \cdots, g_{m} \in E$ and each $m \in N$, showing $\varphi$ to be a fully coherent state on $\mathscr{W}(E)$.

Proof. See [7], [8], and [9].

For arbitrary initial state $\omega \in \mathscr{F}_{a, F}$ of the radiating system the associated time 
asymptotic photon states $R_{t}(\omega), t \in \mathbb{R}$, and $\bar{R}(\omega)$ are fully coherent and factorize with respect to the linear form $f \in E \mapsto \beta \lambda L(f)$, which is independent of $\omega$ and is given by the macroscopic preparation (expressed by $\eta$ and $\gamma$ via eq. (2.8)), the atomic level-splitting $\varepsilon>0$ and the coupling function $\phi$. Only the phase distribution in $R_{t}(\omega)$ depends on $\omega$ (and contains a periodic time dependence). In any case the phase distribution is positive so that the time asymptotic states are classical.

If the radius $\beta$ of the circle line $T_{\eta, \gamma}$ is zero, that is $|\gamma-1 / 2|=\eta / 2$ by (2.8), then $\mathscr{R}_{a s}=\left\{\omega_{F}\right\}$ consists only of the Fock state. Hence in the following we suppose $\beta \neq 0$, that is, the inequality (2.2) is proper. Now, as mentioned in the introduction, the boundedness resp. unboundedness of the linear form $L: E \rightarrow C$ comes into play.

Proposition 4.2. The following three assertions are equivalent (remember (3.10) and the condition $\phi \in E_{\max }$ ):

(i) $\bar{\phi} \mid S_{\varepsilon}=0$;

(ii) $\phi \in \mathscr{D}\left((\sqrt{-\Delta}-\varepsilon)^{-1}\right)$, that is $\left\|(\sqrt{-\Delta}-\varepsilon)^{-1} \phi\right\|^{2}=\int_{\mathbb{R}^{3}} \frac{|\hat{\phi}(k)|^{2}}{\| k|-\varepsilon|^{2}} \mathrm{~d}^{3} k<\infty$;

(iii) $L: E \rightarrow C$ is bounded (with respect to the norm on $E$ ).

If one of these conditions is fulfilled, then $L^{\prime}=0$ (cf. eq. (3.7)) and the linear form $\mathbb{L}=L^{\prime \prime}$ (cf. eqs. (3.6) and (3.8)) is given by

$$
L(f)=-\left\langle(\sqrt{-\Delta}-\varepsilon)^{-1} \phi \mid f\right\rangle \quad \forall f \in E
$$

Proof. (i) $\Rightarrow$ (ii) Because of $\phi \in E_{\max }$ and $\left.\bar{\phi}\right|_{S_{c}}=0$, for $k \in \mathbb{R}^{3}$ with $|k|=1$ we have $\lim _{x \rightarrow \epsilon} \frac{\hat{\phi}(x k)}{x-\varepsilon}=\left.\frac{\partial \hat{\phi}(x k)}{\partial x}\right|_{x=\varepsilon}$, from which follows $(x, k) \mapsto \frac{\hat{\phi}(x k)}{x-\varepsilon}$ is continuous for $x$ in a neighborhood of $\varepsilon>0$ and for $k$ with $|k|=1$. Hence $x \in\left[0, \infty\left[\mapsto \frac{b \phi(x)}{(x-\varepsilon)^{2}}\right.\right.$, where

$$
b \phi:\left[0, \infty\left[\rightarrow C, x \mapsto \int_{|k|=x}|\hat{\phi}(k)|^{2} \mathrm{~d} S(k)=\int_{|k|=1}|\hat{\phi}(x k)|^{2} x^{2} \mathrm{~d} S(k),\right.\right.
$$


is continuous in a neighborhood of $\varepsilon>0$. Consequently, by splitting $\int_{\mathbb{R}^{3}} \cdots \mathrm{d}^{3} k$ into its radial and spherical integrals the assertion (ii) follows:

$$
\left\|(\sqrt{-\Delta}-\varepsilon)^{-1} \phi\right\|^{2}=\int_{0}^{\infty} \frac{b_{\phi}(x)}{|(x-\varepsilon)|^{2}} \mathrm{~d} x<\infty .
$$

(ii) $\Rightarrow$ (i),(iii) Assume $\left.\hat{\phi}\right|_{S_{\varepsilon}} \neq 0$. Then $b \phi(\varepsilon) \neq 0$. Hence by the continuity of $b_{\phi}$ there exist $\alpha, \delta>0$ with $b \phi(x) \geq \delta \forall x \in[\varepsilon-\alpha, \varepsilon+\alpha]$. Because of $0<\delta^{2}$ $\frac{1}{(x-\varepsilon)^{2}} \leq \frac{b \phi(x)}{(x-\varepsilon)^{2}} \forall x \in[\varepsilon-\alpha, \varepsilon+\alpha]$, and since $x \mapsto(x-\varepsilon)^{-2}$ is not an element of $\mathrm{L}^{1}([\varepsilon-\alpha, \varepsilon+\alpha])$ we have a contradiction to (4.3). Consequently $\left.\bar{\phi}\right|_{S_{\varepsilon}}=0$, and thus $L^{\prime}=0$ and $L(f)=-\left\langle(\sqrt{-\Delta}-\varepsilon)^{-1} \phi \mid f\right\rangle f \in E$, the boundedness of $L$.

(iii) $\Rightarrow$ (ii) Clearly $E_{\varepsilon}:=\mathbb{F}^{-1}\left(\mathscr{C}_{c, 0, \epsilon}^{\infty}\left(\mathbb{R}^{3}\right)\right)$ is a subset of $\mathscr{D}\left((\sqrt{-\Delta}-\varepsilon)^{-1}\right)$. Since $E_{\varepsilon}$ is also invariant with respect to the unitary group $\exp \left\{i t(\sqrt{-\Delta}-\varepsilon)^{-1}\right\}$ (which is shown by Fourier transformation), by [19, Theorem VIII.11] $E_{\varepsilon}$ is a core for $(\sqrt{-\Delta}-\varepsilon)^{-1}$

$L$ being bounded, there is an $h \in \mathrm{L}^{2}\left(\boldsymbol{R}^{3}\right)$ with $L(f)=\langle h \mid f\rangle \forall f \in E$. Because of $E_{\varepsilon} \subseteq E$ we have $\langle h \mid f\rangle=-\left\langle\phi \mid(\sqrt{-\Delta}-\varepsilon)^{-1} f\right\rangle \forall f \in E_{\varepsilon}$. Since $E_{\varepsilon}$ is a core for $(\sqrt{-\Delta}-\varepsilon)^{-1}$, by the definition of the adjoint it follows that $\phi$ is an element of the domain of the selfadjoint operator $(\sqrt{-\Delta}-\varepsilon)^{-1}$. Hence $h=-(\sqrt{-\Delta}-\varepsilon)^{-1} \phi$.

Remark 4.3. In the case of $F^{-1}\left(\mathscr{C}_{c, 0}^{\infty}\left(\mathbb{R}^{3}\right)\right) \subseteq E \subseteq E_{\max }$ and $\left.\hat{\phi}\right|_{S_{\varepsilon}} \neq 0$, by similar methods as in the above proposition one proves that the linear form $a L^{\prime}+b L^{\prime \prime}: E \rightarrow C$ is unbounded for all tupels $(a, b) \in \mathbb{C}^{2} \backslash\{(0,0)\}$.

Since the coupling function $\phi \in E_{\max }$ is uniquely determined by the wave functions of the two energy levels of the atoms or molecules under consideration (remember: level-splitting $\varepsilon>0$ ), in physical applications in general one has $\left.\hat{\phi}\right|_{S_{\varepsilon}} \neq 0$, which is equivalent to the unboundedness of $L: E \rightarrow C$.

\subsection{Destruction and revival of coherence}

The set of all (fully) coherent Fock-normal states on $\mathscr{W}(E)$, which are 
discussed by Glauber to be of physical relevance [20], one obtains by considering in eq. (3.16) those measures $\mu \in M_{+}^{1}\left(\mathrm{~L}^{2}\left(\mathbb{R}^{3}\right)\right)$ which are concentrated on the unit circle $\mathbb{T} g$ for $g \in \mathrm{L}^{2}\left(\boldsymbol{R}^{3}\right)$, where $\mathbb{T}=\left\{\mathrm{e}^{i, 9} \in \mathbb{C} \mid \vartheta \in[0,2 \pi[\}\right.$ is the one-dimensional torus.

Proposition 4.4. Let us start at time $t=0$ with a product state $\omega_{a} \otimes \omega_{F}^{\mu} \in \mathscr{F}_{a, F}$ with arbitrary $\omega_{a} \in \mathscr{F}_{a}$ and $\mu \in M_{+}^{1}(\mathrm{Tg})$ for some $g \in \mathrm{L}^{2}\left(\mathbb{R}^{3}\right)$. Then $\left.v_{t}\left(\omega_{a} \otimes \omega_{F}^{\mu}\right)\right|_{b}$ is (fully) coherent for some $t>0$, if and only if $g=0$, that is, if and only if $\mu$ is the point measure at zero, or equivalently if and only if $\omega_{F}^{\mu}=\omega_{F}$ is the Fock state. In this case the states $\left.v_{t}\left(\omega_{a} \otimes \omega_{F}\right)\right|_{b}$ are coherent for each time point $t \in \mathbb{R}$ with respect to the bounded linear form $f \in E \mapsto \beta \lambda\left\langle h_{t} \mid f\right\rangle$ (see [7, Definition 2.1] or [8, Definition 1.2]), and their characteristic functions are given by

$$
\begin{aligned}
& \left\langle\left. v_{t}\left(\omega_{a} \otimes \omega_{F}\right)\right|_{b} ; W(f)\right\rangle \\
& =C_{F}(f) \int_{[0,2 \pi[} \exp \left\{i \sqrt{2} \beta \lambda \operatorname{Re}\left(\mathrm{e}^{i \theta}\left\langle h_{t} \mid f\right\rangle\right)\right\} \mathrm{d}\left({\tilde{\rho_{\omega_{a}}}}_{\varphi^{\prime}} \circ \varphi_{-t}^{\eta, \gamma}(\theta)\right) .
\end{aligned}
$$

Proof. Immediate consequence of Proposition 3.4 and [7], [8].

In both cases of the above proposition, $g=0$ and $g \neq 0$, the "wrong" coherence at $t=0$, which is associated with the linear form $f \in E \mapsto\left\langle\frac{i}{\sqrt{2}} g \mid f\right\rangle$ is destroyed during the time evolution. After enough time having elapsed $(t \rightarrow \infty)$ the new quantum optical coherence is build up, which now is in accordance with the resonance frequency of the atoms and expressed by the factorization of (4.1) in terms of the linear form $L$.

\subsection{Mean number of emitted photons}

Since $L$ is unbounded for $\beta \neq 0$ the time asymptotic states $\mathscr{R}_{\text {as }}$ correspond formally to strictly infinite mean numbers of photons. However, for finite times $t \geq 0$ the states $\left.v_{t}(\omega)\right|_{b}, \omega \in \mathscr{F}_{a, F}$, are normal to the Fock representation and the photon number is finite. Here we investigate, how the photon number increases in time and finally becomes infinite as $t \rightarrow \infty$. We do not calculate the general problem, but start with the case where no photon is present at $t=0$, more exactly, with the initial state $\omega_{a} \otimes \omega_{F} \in \mathscr{F}_{a, F}$, where 
$\omega_{a} \in \mathscr{F}_{a}$ is an arbitrary atomic state.

By means of (4.4), (3.16) and (3.17) for each $t \in R$ the state $\left.v_{t}\left(\omega_{a} \otimes \omega_{F}\right)\right|_{b}$ is given in the Fock representation by the density operator

$$
\begin{aligned}
& \varrho_{t}^{\omega_{a} \otimes \omega_{F}} \\
& \quad=\int_{[0,2 \pi[}\left|W_{F}\left(-i \mathrm{e}^{-i \vartheta} \sqrt{2} \beta \lambda h_{t}\right) \Omega_{F}\right\rangle\left\langle W_{F}\left(-i \mathrm{e}^{-i \vartheta} \sqrt{2} \beta \lambda h_{t}\right) \Omega_{F}\right| \mathrm{d}\left(\tilde{\rho}_{\omega_{a}} \circ \varphi_{-t}^{\eta \cdot \gamma}(\vartheta)\right) .
\end{aligned}
$$

Observing $\left\langle\mathrm{W}_{F}(h) \Omega_{F} \mid N_{R^{3}} W_{F}(h) \Omega_{F}\right\rangle=\frac{\|h\|^{2}}{2} \forall h \in \mathrm{L}^{2}\left(\boldsymbol{R}^{3}\right)$ with the number operator $N_{\boldsymbol{R}^{3}}$ in the Fock space $F_{+} \equiv F_{+}\left(\mathrm{L}^{2}\left(\boldsymbol{R}^{3}\right)\right)$ we get a result, which is also obtained with the restricted formalism in [5].

Proposition 4.5. For each $t \in \boldsymbol{R}$ and every $\omega_{a} \in \mathscr{F}_{a}$ the photon number expectation value is

$$
\left\langle\left. v_{t}\left(\omega_{a} \otimes \omega_{F}\right)\right|_{b} ; \quad N_{R^{3}}\right\rangle=\operatorname{tr}\left[\varrho_{t}^{\omega_{a} \otimes \omega_{F}} N_{R^{3}}\right]=\beta^{2} \lambda^{2}\left\|h_{t}\right\|^{2}
$$

with $h_{t}$ from formula (3.2).

Thus the growth of the mean photon number is determined by the map $t \in \boldsymbol{R} \mapsto\left\|h_{t}\right\|^{2}$, which turns out to be linear in time, a result which goes back to [5].

Proposition 4.6. The following two assertions are valid:

(a) Assume $\left.\hat{\phi}\right|_{S_{\varepsilon}}=0$. Then $\left\|h_{t}\right\| \leq 2\left\|(\sqrt{-\Delta}-\varepsilon)^{-1} \phi\right\| \forall t \in R$, and $\lim _{t \rightarrow \infty}\left\|h_{t}\right\|=$ $\sqrt{2}\left\|(\sqrt{-\Delta}-\varepsilon)^{-1} \phi\right\| . \quad$ Moreover $\lim _{t \rightarrow \infty}\left\langle h_{t} \mid f\right\rangle=-\left\langle(\sqrt{-\Delta}-\varepsilon)^{-1} \phi \mid f\right\rangle \forall f \in E$.

(b) Assume $\left.\hat{\phi}\right|_{S_{\varepsilon}} \neq 0$. Then there exists a continuous function $\sigma:[0, \infty[\rightarrow \boldsymbol{R}$ and constants $c_{1}, c_{2}>0$ such that $|\sigma(t)| \leq c_{1}+c_{2} \sqrt{t}$ for $t \geq 1$ and that for all $t \in[0, \infty[$

$$
\left\|h_{t}\right\|^{2}=2 \pi\left(\int_{S_{\varepsilon}}|\hat{\phi}(k)|^{2} \mathrm{~d} S(k)\right) t+\sigma(t)
$$


Proof. (a) Using the spectral calculus we obtain

$$
h_{t}=(\exp \{-i t(\sqrt{-\Delta}-\varepsilon)\}-1) \tilde{\phi} \text { with } \tilde{\phi}:=(\sqrt{-\Delta}-\varepsilon)^{-1} \phi,
$$

from which follows $\left\|h_{t}\right\| \leq 2\|\tilde{\phi}\|$ and

$$
\begin{aligned}
\lim _{t \rightarrow \infty}\left\|h_{t}\right\|^{2} & =\lim _{t \rightarrow \infty}\langle\tilde{\phi} \|(2-\exp \{i t(\sqrt{-\Delta}-\varepsilon)\}-\exp \{-i t(\sqrt{-\Delta}-\varepsilon)\}) \tilde{\phi}\rangle \\
& =2\|\tilde{\phi}\|^{2}
\end{aligned}
$$

where we have used (3.1). $\lim \left\langle h_{t}+\tilde{\phi} \mid f\right\rangle=0$ also follows from (3.1). $t \rightarrow \infty$

(b) Using the Fourier transformation on $\mathrm{L}^{2}\left(\mathbb{R}^{3}\right)$ and decomposing the integral over $\mathbb{R}^{3}$ into its radial and spherical parts and introducing $H_{t}(x):=\sqrt{b_{\phi}(x)} \frac{\exp \{i(\varepsilon-x) t\}}{\varepsilon-x}, x \in\left[0, \infty\left[\right.\right.$, where $b_{\phi}$ is from (4.2), we obtain $\left\|h_{t}\right\|^{2}=\int_{0}^{t}\left|H_{t}(x)\right|^{2} \mathrm{~d} x$. Since $\left.\hat{\phi}\right|_{s_{\varepsilon}} \neq 0$ we have $b_{\phi}(\varepsilon) \neq 0$ and $\sqrt{b_{\phi}}$ is $\mathscr{C}^{1}$ in a neighborhood of $\varepsilon>0$. With these prerequisites the proof of [5, Theorem 4.6] is applicable and yields the result.

Observe $\int_{S_{\varepsilon}}|\hat{\phi}(k)|^{2} \mathrm{~d} S(k)=\frac{1}{i \pi} L^{\prime}(\phi)$ with the linear form $L^{\prime}$ from (3.7).

For large times by Proposition 4.6 the emission rate is constant and uniqely determined by the coupling constant $\bar{\phi}(k)$ at the resonant modes $k \in S_{\varepsilon}$.

\subsection{Spatial distribution of the emitted photons}

For $\varphi \in \mathscr{R}_{\mathrm{as}}$ in the case of $\beta \neq 0$ and of unbounded $L$ the mean photon number is strictly infinite, however, as we will show, with a finite, non-vanishing density. We follow ideas and methods of [21] and [6].

Assume the one-photon testfunction space $E$ such that $\mathrm{L}^{2}(U) \cap E=: \mathbb{E}_{U}$ is dense in $L^{2}(U)$ for each open and bounded subset $U \subset \mathbb{R}^{3}$. Thus the Weyl algebra $\mathscr{W}(E)$ has a quasilocal substructure:

$$
\overline{\bigcup_{U} \mathscr{W}\left(E_{U}\right)}=\mathscr{W}\left(E^{c}\right) \subseteq \mathscr{W}(E), \text { where } E^{c}:=\bigcup_{U} E_{U}
$$


The coupling function $\phi \in E_{\max }$ we suppose to satisfy $\frac{\hat{\phi}}{|\cdot|+1} \in \mathrm{L}^{1}\left(\boldsymbol{R}^{3}\right)$. By use of the Fourier transformation for the linear form $L$ from Proposition 3.1 with Fubini's theorem and Lebesgue's dominated convergence we obtain for all $f \in E^{c}$

$$
L(f)=\int_{\boldsymbol{R}^{3}} \overline{L(x)} f(x) \mathrm{d}^{3} x
$$

with the bounded and continuous function $L: R^{3} \rightarrow C$ given by

$$
L(x)=-(2 \pi)^{-3 / 2}\left\{i \pi \int_{S_{\varepsilon}} \hat{\phi}(k) \mathrm{e}^{i k \cdot x} \mathrm{~d} S(k)-\mathrm{pv} \int_{\mathbb{R}^{3}} \frac{\hat{\phi}(k) \mathrm{e}^{i k \cdot x}}{|k|-\varepsilon} \mathrm{d}^{3}(k)\right\} .
$$

Clearly the function $L: R^{3} \rightarrow C$ should be decomposed in terms of the Fourier transform $\hat{T}$ (cf. [22] p. 5) of the tempered distribution $T$ on the space $\mathscr{S}\left(\boldsymbol{R}^{3}\right)$ of function with rapid decrease,

$$
T(\psi)=i \pi \int_{S_{\varepsilon}} \psi(k) \mathrm{d} S(k)-\mathrm{pv} \int_{\boldsymbol{R}^{3}} \frac{\psi(k)}{|k|-\varepsilon} \mathrm{d}^{3}(k), \quad \psi \in \mathscr{S}\left(\boldsymbol{R}^{3}\right) .
$$

Under the condition for the coupling function $\phi \in \mathscr{S}\left(\boldsymbol{R}^{3}\right)$ by use of [22, Theorem IX.4] one easily checks that $L$ is an infinitely differentiable function, which together with its derivatives is polynomially bounded and given by the convolution

$$
L(x)=(2 \pi)^{-3 / 2} \overline{(\hat{T} \star \bar{\phi})(x)} \quad \forall x \in \boldsymbol{R}^{3} .
$$

However, here in the context of distribution theory (4.5) is valid for all $f \in \mathscr{S}\left(\boldsymbol{R}^{3}\right)$. And (4.6) may be expressed as

$$
\overline{L(x)}=T(\bar{\phi} \exp \{-i\langle. \mid x\rangle\}) \quad \forall x \in R^{3},
$$

a result which one could also obtain from [22, Theorem IX.5].

Let us return from distribution theory to the above case $\phi \in E_{\max }$ with $\frac{\hat{\phi}}{|\cdot|+1} \in \mathrm{L}^{1}\left(\boldsymbol{R}^{3}\right)$ and (4.5) to be valid for all $f \in E^{c}$. Obviously $L \in \mathrm{L}^{2}{ }_{\text {loc }}\left(\boldsymbol{R}^{3}\right)$ is 
locally square integrable, from which follows that the restriction of the linear from $L: E \rightarrow \mathbb{C}$ to $E_{U}$ is bounded: $L(f)=\left\langle L_{U} \mid f\right\rangle \forall f \in E_{U}$, where $L_{U}(x)=L(x)$ for $x \in U$ and $L_{U}(x)=0$ for $x \notin U$. As a consequence, for each $\omega \in \mathscr{F}_{a, F}$ the time asymptotic states $R_{t}(\omega)$ and $\bar{R}_{\eta, \gamma}$ are locally Fock-normal. More exactly: For $U \subset \mathbb{R}^{3}$ open and bounded, the restricted states $\left.R_{t}(\omega)\right|_{\mathscr{W}\left(E_{U}\right)}$ and $\left.\bar{R}_{\eta, \gamma}\right|_{\mathscr{W}\left(E_{U}\right)}$ are normal to the Fock representation $\Pi_{F}$ of the local Weyl algebra $\mathscr{W}\left(E_{U}\right)$ on the local Bose-Fock space $F_{+}\left(\mathrm{L}^{2}(U)\right)$, [8, Proposition 2.5]. From (3.16) and (3.17) we obtain

$$
\left\langle R_{t}(\omega) ; Y\right\rangle=\operatorname{tr}\left[\varrho_{U, t}^{\omega} \Pi_{F}(Y)\right], \quad\left\langle\bar{R}_{\eta, \gamma} ; Y\right\rangle=\operatorname{tr}\left[\bar{\varrho}_{U}^{\omega} \Pi_{F}(Y)\right] \quad \forall Y \in \mathscr{W}\left(E_{U}\right)
$$

with the density operators $\varrho_{U, t}^{\omega}$ and $\bar{\ell}_{U}^{\omega}$ on $F_{+}\left(\mathrm{L}^{2}(U)\right)$ given by

$$
\varrho_{U, t}^{\omega}=\int_{[0,2 \pi[}\left|W_{F}\left(-i \mathrm{e}^{-i \vartheta} \sqrt{2} \beta \lambda L_{U}\right) \Omega_{F}\right\rangle\left\langle W_{F}\left(-i \mathrm{e}^{-i \vartheta} \sqrt{2} \beta \lambda L_{U}\right) \Omega_{F}\right| \mathrm{d}\left(\rho_{\omega} \circ \varphi_{-t}^{\eta, \gamma}(\vartheta)\right)
$$

resp. $\bar{\varrho}_{U}^{\omega}$ in the same way, however with the normalized Lebesque measure $\lambda^{\eta, \gamma}$. Analogously to Proposition 4.5 we get:

Proposition 4.7. Let be $N_{U}$ the number operator in the local Fock space $F_{+}\left(\mathrm{L}^{2}(U)\right)$. It follows for each $\omega \in \mathscr{F}_{a, F}$ and $t \in \mathbb{R}$

$$
\left\langle R_{t}(\omega) ; N_{U}\right\rangle=\left\langle\bar{R}_{\eta, \gamma} ; N_{U}\right\rangle=\beta^{2} \lambda^{2}\left\|\mathrm{~L}_{U}\right\|^{2}=\beta^{2} \lambda^{2} \int_{U}|L(x)|^{2} \mathrm{~d}^{3} x
$$

As a consequence of Proposition 4.7 the photon density is given with the integral kernel $L: \mathbb{R}^{3} \rightarrow C$ of (4.5) resp. (4.6) according to

$$
x \in \boldsymbol{R}^{3} \mapsto \beta^{2} \lambda^{2}|L(x)|^{2}
$$

for each time asymptotic state $\varphi \in \mathscr{R}_{\text {as }}$. Since the linear form $L: E \rightarrow C$ is unbounded we have $L \notin \mathrm{L}^{2}\left(\mathbb{R}^{3}\right)$, which also implies the total number of photons in all of $\boldsymbol{R}^{3}$ to be strictly infinite.

\section{$\S 5$. The Case of Arbitrary Polarization}

The quantization procedure in the Coulomb gauge leads to the $C^{*}$-Weyl 
algebra $\mathscr{W}(\widetilde{E})$ over a testfunction space $\widetilde{E}$ dense in

$$
\mathrm{L}_{\text {div }}^{2}\left(\boldsymbol{R}^{3}, \boldsymbol{C}^{3}\right)=\left\{f=\left(f_{1}, f_{2}, f_{3}\right) \in \mathrm{L}^{2}\left(\boldsymbol{R}^{3}, \boldsymbol{C}^{3}\right) \mid \nabla \cdot f=0\right\}
$$

as algebra of observables for the photons. Here $\nabla \cdot f=0$ is meant in the distributional sense: $0=(\nabla \cdot f)(\psi)=-\langle f \mid \operatorname{grad} \psi\rangle \forall \psi \in \mathscr{S}\left(\boldsymbol{R}^{3}\right)$.

Defining the Fourier transformation $F: f \mapsto \hat{f}$ on $\mathrm{L}^{2}\left(\boldsymbol{R}^{3}, \boldsymbol{C}^{3}\right)$ componentwise the condition $\nabla \cdot f=0$ is equivalent to $k \cdot \hat{f}(k)=0$ for almost all $k \in \mathbb{R}^{3}$. In momemtum space the orthogonal projection from $\mathrm{L}^{2}\left(\boldsymbol{R}^{3}, C^{3}\right)$ onto $F\left(\mathrm{~L}^{2}\right.$ div $\left.\left(\boldsymbol{R}^{3}, \boldsymbol{C}^{3}\right)\right)$ is performed with the linear map $P_{\text {div }}$ given on each function $g: R^{3} \rightarrow C^{3}$ by

$$
P_{\mathrm{div}} g: \boldsymbol{R}^{3} \rightarrow C^{3}, k \mapsto g(k)-\frac{k \cdot g(k)}{|k|^{2}} k
$$

Let us turn to the one-photon dynamics. For the Laplacian $\tilde{\Delta}$ in $\mathrm{L}^{2}\left(\boldsymbol{R}^{3}, \boldsymbol{C}^{3}\right)$, which also is defined componentwise, $\tilde{\Delta} f=\left(\Delta f_{1}, \Delta f_{2}, \Delta f_{3}\right)$ for $f_{j} \in \mathscr{D}(\Delta)$, we have $\left(\boldsymbol{F} \mathrm{e}^{-i t \tilde{\Delta}_{f}} f\right)(k)=\mathrm{e}^{i t|k|^{2}} \hat{f}(k)$ for almost all $k \in \boldsymbol{R}^{3}$, from which follows $\nabla \cdot\left(\mathrm{e}^{-i t \tilde{\Delta}} f\right)=0$, if and only if $\nabla \cdot f=0$. Thus $\mathrm{e}^{i \tau \tilde{\Delta}}$ for each $t \in \boldsymbol{R}$ leaves $\mathrm{L}^{2}{ }_{\text {div }}\left(\boldsymbol{R}^{3}, \boldsymbol{C}^{3}\right)$ invariant. Consequentely $\mathrm{L}^{2}{ }_{\text {div }}\left(\boldsymbol{R}^{3}, \boldsymbol{C}^{3}\right)$ is a reducing subspace for $\tilde{\Delta}$ [23, Theorem 7.2.8], and the restriction of $\tilde{\Delta}$ from $\mathrm{L}^{2}\left(\boldsymbol{R}^{3}, C^{3}\right)$ to $\mathrm{L}_{\text {div }}^{2}\left(\boldsymbol{R}^{3}, \boldsymbol{C}^{3}\right)$ is a selfadjoint operator in $\mathrm{L}_{\text {div }}^{2}\left(\boldsymbol{R}^{3}, \boldsymbol{C}^{3}\right)$, which we denote also by $\tilde{\Delta}$. Clearly, for each Borel function $u: R \rightarrow C$ the operator $u(-\tilde{\Delta})$ on $\mathrm{L}^{2}\left(\boldsymbol{R}^{3}, \boldsymbol{C}^{3}\right)$ resp. $\left.\mathrm{L}_{\text {div }}{ }^{2} \boldsymbol{R}^{3}, \boldsymbol{C}^{3}\right)$ works componentwise and its Fourier transform $\boldsymbol{F} u(-\tilde{\Delta}) \boldsymbol{F}^{-1}$ is just the componentwise multiplication with $k \in \boldsymbol{R}^{3} \mapsto u\left(\|k\|^{2}\right)$.

The one-photon Hamiltonian is given by $S:=\sqrt{-\widetilde{\Delta}}$ (cf. [2, Section 3.1]), which is selfadjoint in $\mathrm{L}_{\text {div }}^{2}\left(\boldsymbol{R}^{3}, C^{3}\right)$, and the testfunction space $\widetilde{E}$ should be invariant under the unitary one-photon dynamics $\mathrm{e}^{i t S}, t \in \boldsymbol{R}$.

From (5.1) is easily seen that $P_{\text {div }}$ commutes with $F \mathrm{e}^{i t \sqrt{\bar{\Delta}}} F^{-1}=\mathrm{e}^{i t|\cdot|^{2}}$ and that $P_{\text {div }}\left(\mathscr{C}_{c, 0, \epsilon}^{\infty}\left(R^{3}, C^{3}\right)\right)=\mathscr{C}_{c, 0, \epsilon}^{\infty}\left(R^{3}, C^{3}\right) \cap F\left(\mathrm{~L}_{\text {div }}^{2}\left(R^{3}, C^{3}\right)\right)$ is dense in $\mathbb{F}\left(\mathrm{L}^{2}{ }_{\text {div }}\left(\boldsymbol{R}^{3}, \boldsymbol{C}^{3}\right)\right)$. A similar result holds for $\mathscr{C}_{c, 0}^{\infty}\left(\boldsymbol{R}^{3}, \boldsymbol{C}^{3}\right)$. In this way one immediately constructs appropriate testfunction spaces $\widetilde{E}$ with

$$
F^{-1}\left(\mathscr{C}_{c, 0, \epsilon}^{\infty}\left(\boldsymbol{R}^{3}, \boldsymbol{C}^{3}\right)\right) \subseteq \widetilde{E} \subseteq \widetilde{E}_{\max }
$$

(cf. eq. (3.10)), where (cf. eq. (3.5))

$$
\widetilde{E}_{\max }:=F^{-1} P_{\mathrm{div}} F\left(\bigoplus_{j=1}^{3} E_{\max }\right)
$$




$$
=\left\{f \in \mathbb{L}_{\text {div }}^{2}\left(\mathbb{R}^{3}, C^{3}\right) \mid \hat{f} \text { is } \mathscr{C}^{1} \text { in a neighborhood of } S_{\varepsilon}\right\} .
$$

From this it is clear, that - by working componentwise-all arguments are analogous to the case of one direction of polarization. For example, with the coupling function $\phi \in \widetilde{E}_{\max }$ the limiting linear form $L: \widetilde{E} \rightarrow \mathbb{C}$ from (3.3) is obtained similar to Proposition 3.1 as

$$
L(f)=i \pi \int_{S_{\varepsilon}} \overline{\phi(k)} \cdot \hat{f}(k) \mathrm{d} S(k)-\mathrm{pv} \int_{\mathbb{R}^{3}} \frac{\bar{\phi}(k) \cdot \hat{f}(k)}{|k|-\varepsilon} \mathrm{d}^{3} k \quad \forall f \in \widetilde{E} .
$$

In terms of the smeared classical vector field $L(f)$ the emission characteristics of the emitted radiation can be obtained. For notational simplicity we omit the representation indices for the annihilation and creation operators. In terms of an (arbitrary) orthonormal basis $\left\{e_{n} \mid n \in \mathbb{N}_{0}\right\}$ in $\mathbb{L}_{\text {div }}^{2}\left(\mathbb{R}^{3}, \mathbb{C}^{3}\right)$ consisting of real-valued functions $\in_{n}: \mathbb{R}^{3} \rightarrow \mathbb{R}^{3}$, which are smooth enough, one obtains the operator of the Poynting vector $\mathbf{S}(x)=\mathbb{E}(x) \times \mathbb{B}(x)$ at $x \in \mathbb{R}^{3}$ (cross product of the electric and the magnetic field)

$$
\begin{aligned}
\mathbf{S}(x)= & \frac{c^{2} \hbar}{2} \sum_{m, n=0}^{\infty} \mathbf{e}_{m}(x) \times\left(\nabla \times \mathbf{e}_{n}(x)\right) \\
& \times\left[a\left(S^{1 / 2} \mathbf{e}_{m}\right) a\left(S^{1 / 2} \mathbf{e}_{n}\right)+a^{*}\left(S^{-1 / 2} \mathbf{e}_{n}\right) a\left(S^{1 / 2} \mathbf{e}_{m}\right)\right. \\
& \left.\quad-a^{*}\left(S^{1 / 2} \mathbf{e}_{m}\right) a\left(S^{-1 / 2} \mathbf{e}_{n}\right)-a^{*}\left(S^{1 / 2} \mathbf{e}_{m}\right) a^{*}\left(S^{-1 / 2} \mathbf{e}_{n}\right)\right]
\end{aligned}
$$

where the annihilation and creation operators are normally ordered, [2, Section 3.1]. Using the factorization (4.1) and the integral kernel $x \in \mathbb{R}^{3} \mapsto L(x) \in \mathbb{C}^{3}$ (similar to equation (4.5)) the expectation values of $\mathrm{S}(x)$ for the time asymptotic averaged states are calculated [2, Section 7.5]

$$
\left\langle\bar{R}_{\eta, \gamma} ; \mathbf{S}(x)\right\rangle=\lambda^{2} \beta^{2} \operatorname{Im}\left(\overline{S^{1 / 2} L(x)} \times\left(\nabla \times S^{-1 / 2} L(x)\right)\right) \quad \forall x \in \mathbb{R}^{3} \backslash\{0\}
$$

Here $S^{ \pm 1 / 2} L$ means the formal application of the operator $S^{ \pm 1 / 2}$ (obtained from the functional calculus) to the function $L$, which, however, is not an 
element of its domain, and given by

$$
\begin{gathered}
S^{1 / 2} L(x):=\int_{\mathbb{R}^{3}}|k|^{1 / 2} \hat{\phi}(k) \mathrm{e}^{i k \cdot x} \delta^{+}(\|k\|-\varepsilon) \mathrm{d}^{3} k, \\
\nabla \times S^{-1 / 2} L(x):=\int_{\mathbb{R}^{3}} \frac{k \times \hat{\phi}(k)}{|k|^{1 / 2}} \mathrm{e}^{i k \cdot x} \delta^{+}(\|k\|-\varepsilon) \mathrm{d}^{3} k,
\end{gathered}
$$

where $\delta^{+}(\|k\|-\varepsilon)$ denotes the distribution $T$ from (4.7).

The formula demonstrates the dependence of the emission characteristics from the wave functions of the selected two energy levels of the considered type of atoms and molecule, which determine the coupling function $\phi$ uniquely. For the example of hydrogenlike atoms the coupling function $\phi \in \widetilde{E}_{\max }$ is calculated as follows: With the wave functions $\Psi_{\uparrow}, \Psi_{\downarrow} \in \mathrm{L}^{2}\left(\boldsymbol{R}^{3}\right)$ for the upper resp. lower energy level of the active electron (level-splitting $\varepsilon>0$ ) the Fourier transform $\hat{\phi}$ is proportional to $P_{\mathrm{div}} \frac{F_{\downarrow \uparrow}}{|\cdot|^{1 / 2}}$, (cf. [2], [3]) where the function $F_{\downarrow \uparrow}$ is given by the scalar products

$$
F_{\downarrow \uparrow}: R^{3} \rightarrow C^{3}, k \mapsto\left\langle\Psi_{\downarrow} \mid\left(p \mathrm{e}^{-i k \cdot q}+\mathrm{e}^{-i k \cdot q} p\right) \Psi_{\uparrow}\right\rangle .
$$

Here $p=-i\left(\frac{\partial}{\partial x_{1}}, \frac{\partial}{\partial x_{2}}, \frac{\partial}{\partial x_{3}}\right)$ denotes the usual momentum operator and $q=\left(x_{1}, x_{2}, x_{3}\right)$ the position operator in the Hilbert space $\mathrm{L}^{2}\left(\boldsymbol{R}^{3}\right)$ for the electron. For the interaction is used the rotating-wave-approximation. Concrete calculations of $\phi(1 s-2 s$ and $1 s-2 p)$ and estimates for $\hat{\phi}$ are provided in [3].

\section{Acknowledgements}

One of us (R.H.) is grateful to the Deutsche Forschungsgemeinschaft for financial support.

\section{References}

[ 1 ] Honegger, R., Rieckers, A. and Unnerstall, Th., Perturbational Dynamics of the Infinite Dicke Model, Z. Naturforsch., 48a (1993), 1043-1053.

[2 ] Honegger, R., Globale Quantentheorie der Strahlung, Tübingen, Thesis, 1991.

[ 3 ] Pfeifer, P., Chiral Molecules - a Superselection Rule induced by the Radiation Field, 
Dissertation ETH No. 6551, ok Gotthard $S+D A G$, Zürich, 1980.

[ 4 ] Honegger, R., The Dynamical Generation of Macroscopic Coherent Light. Proceedings of NATO ASI, Large-Scale Molecular Systems: Quantum and Stochastic Aspects. Beyond the Simple Molecular Picture, Maratea, Italy, 1990. Eds. W. Gans, A. Blumen and A. Amann, Plenum Press, New York 1991.

[ 5 ] Davis, E.B., Exact Dynamics of an Infinite-Atom Dicke Maser Model I. Commun. Math. Phys., 33, (1973), 187-205.

[6] - The Infinite Atom Dicke Maser Model II, Commun. Math. Phys., 34 (1973), 237-249.

[ 7 ] Honegger, R. and Rapp, A., General Glauber coherent states on the Weyl algebra and their phase integrals, Physica A, 167 (1990), 945-961.

[ 8 ] Honegger, R. and Rieckers, A., The general form of non-Fock coherent boson states, Publ. RIMS, Kyoto Univ., 26 (1990), 397-417.

[ 9 ] —, First order coherent boson states, Helv. Phys. Acta, 65 (1992), 965-984.

[10] - On higher order coherent states on the Weyl algebra, Lett. Math. Phys., 24 (1992), 221-225.

[11] Honegger, R., The general form of the microscopic coherent boson states, Physica $A$, 198 (1993), 179-209.

[12] Glauber, R.J., The Quantum Theory of Optical Coherence, Phys. Rev., 130 (1963), 2529-2539, Coherent and Incoherent States of the Radiation Field, Phys. Rev., 131 (1963), 2766-2788, in Quantum Optics and Electronics, Les Houches 1964, edited by C. de Witt, A. Blandin and C. Cohen-Tannoudji Gordon and Breach, New York: 1965.

[13] Andreev, A.V., Emel'yanov, V.I. and Il'inskii, Yu.A., Collective Spontaneous Emission, Sov. Phys. Usp., 23 (1980), 493-514.

[14] Bratteli, O. and Robinson, D.W., Operator Algebras and Quantum Statistical Mechanics, Vol. II. Springer, Berlin, Heidelberg, New York, 1981.

[15] Amrein, W.O., Non-Relativistic Quantum Dynamics, D. Reidel Publishing Company, Dordrecht Holland, 1981.

[16] Schwartz, L., Mathematische Methoden der Physik I, B.I.-Wissenschaftsverlag, Mannheim, 1974.

[17] Forster, O., Analysis 3, Vieweg, Braunschweig, 1981.

[18] Davies, E.B., Quantum Theory of Open Systems, Academic Press, London, New York, San Francisco, 1976.

[19] Reed, M. and Simon, B., Methods of Modern Mathematical Physics I; Functional Analysis, Academic Press, New York, London, Toronto, Sydney, San Francisco, 1980.

[20] Titulaer, U.M. and Glauber, R.J., Density Operators for Coherent Fields, Phys. Rev., 145 (1966), 1041-1050.

[21] Araki, H. and Woods, E.J., Representations of the Canonical Commutation Relations Describing a Nonrelativistic Infinite Free Bose Gas, J. Math. Phys., 4 (1963), 637-662.

[22] Reed, M. and Simon, B., Methods of Modern Mathematical Physics II; Fourier Analysis, Self-Adjointness, Academic Press, New York, London, Toronto, Sydney, San Francisco, 1975.

[23] Weidmann, J., Linear Operators in Hilbert Spaces, Springer-Verlag, Berlin, Heidelberg, New York, 1980. 